Jürgen Eppelsheim (München)

\title{
Pommern tiefer Lage (Bombardi, Bombardoni, Bomharte) als Bestandteil des Basso-continuo-Instrumentariums
}

\author{
William Waterhouse in gratam memoriam
}

Johann Christoph Altnickols (1719-1759) Osterkantate Ich lebe und ihr sollt auch leben hat nach dem Titelblatt der handschriftlichen Quelle ${ }^{1}$ folgende Besetzung: „Clarino 3. ${ }^{2}$ / Timpano. / Oboe 2. / Violino 2. / Viola. / Violoncello. / Soprano. Alto. Tenore. Basso. / con Organo“. Detaillierteren Aufschluss gibt die Bezeichnung der einzelnen Stimmen: So heißt dort die dritte Trompete nicht Clarino, sondern „Principal“, entsprechend der insgesamt tieferen Lage der Partie, die den 9. Naturton $\left(d^{2}\right)$ nicht überschreitet, dagegen den 3. Naturton $(g)$ sehr häufig fordert. Die mit „Violoncello e Bassono“ bezeichnete Stimme zeigt an, dass außer dem erstgenannten Instrument als Bass des instrumentalen Ensembles im engeren Sinne auch ein Fagott der neueren Art beteiligt war. ${ }^{3}$ Die Orgel („Organo“), abgesehen von der „Aria Furioso“ Nr. 6, für welche „senza Organo“ gilt, Klangbasis des Gesamtensembles, wurde nach Ausweis zweier weiterer (im Titelblatt gleich dem „Bassono“ nicht erscheinender) Instrumentalstimmen von „Violono“4 (der auch an Nr. 6 teilnimmt) und „Bombardo“ unterstützt. ${ }^{5}$

Die zur Entstehungszeit der Kantate in Deutschland noch relativ neuen, aber seit ihrer dortigen Einführung zu Beginn des Jahrhunderts rasch zu großer Bedeutung gelangten französischen Holzblasinstrumente Oboe (Hautbois) und Fagott (Basson) hatten im Bereich kirchlicher Figuralmusik eine neue Situation geschaffen: Ihre die Klangnatur wesentlich mitprägende Einstimmung im „Kammerton“ war um zwei Halbtöne („hoher Kammerton“) oder sogar drei Halbtöne tiefer („tiefer Kammerton“) als der über Jahrhunderte etablierte (und bis ins mittlere 19. Jahrhundert in Gebrauch bleibende) „Chorton“ der Or-

1 Abschrift (Direktionsstimme und vollständiger Stimmensatz) in der Biblioteka Gdánska Polskiej Akademii Nauk, Danzig. Alle auf diese Quelle bzw. auf Altnickols Kantate überhaupt bezogenen Äußerungen des vorliegenden Beitrags gründen sich auf die von Rainer Goede edierte und mit Kommentar und Kritischem Bericht versehene Partiturausgabe, Ansbach: Edition Archiv (2003).

2 Diese Ziffer bezieht sich auf die Anzahl solcher Instrumente bzw. der ihnen zugeordneten Partien; entsprechendes gilt für Oboen und Violinen.

3 Aus seinem Herkunftsland hatte dieses (im heutigen Sprachgebrauch zur Unterscheidung vom älteren Dulzian als Barockfagott bezeichnete) Instrument den französischen Namen Basson nach Deutschland mitgebracht, der des Öfteren, wenn auch unzutreffend, zu Bassono italianisiert wurde (so auch von J. S. Bach).

4 Auch dieses Produkt einer gewissen Sorglosigkeit in sprachlicher Hinsicht findet sich gelegentlich. Die seit einiger Zeit ohne Not dornig gewordene Diskussion um die Wortbedeutung(en) von „Violone“ soll hier nicht aufgenommen werden (es wäre m. E. hilfreich, der sprachlichen Substanz nahe zu bleiben, indem man dieses Wort recht allgemein als „große Viola“ erläutert, „Viola“ wiederum, auf sicherer Quellenbasis früher Zeit, als Streichinstrument generell). Als gewiss erscheint im vorliegenden Falle eines das Violoncello enthaltenden Instrumentariums, dass der Violone als 16', in der Unteroktave der notierten Töne, erklingen soll.

5 Der „Bombardo“ spielt in den Orchesterritornellen der eröffnenden „Aria“, im „Tutti“ von Chor und Orchester, Nr. 3 (ohne die Piano-Abschnitte Takt 62-71 und 79-98) und im Schlusschoral Nr. 7. 
gel. ${ }^{6}$ Als Lösung für ein Zusammenwirken bot sich an, die Komposition insgesamt nach wie vor am Chorton der Orgel auszurichten, Oboen- und Fagottstimmen aber um zwei bzw. drei Halbtöne höher zu transponieren. Dieses Verfahren wandte auch J. S. Bach in seinen vor dem Leipziger Amtsantritt entstandenen Kirchenstücken an. In Leipzig dagegen war schon von seinem Amtsvorgänger Kuhnau ein anderes Verfahren eingeführt worden: Die Komposition orientierte sich nunmehr am („hohen“) Kammerton, der für das gesamte vokale wie instrumentale Ensemble galt, ${ }^{7}$ während die Continuo-Stimme für die Orgel um zwei Halbtöne nach unten zu transponieren war.

In leichter Abänderung gilt diese neuere Praxis auch für Altnickols Osterkantate. Singstimmen, Streicher (außer Violone) und Oboen (nebst „Bassono“, der ja mit dem Violoncello aus gemeinsamer Stimme spielte) musizieren im („hohen“) Kammerton, die beiden mit der Orgel gleichlaufenden Instrumente Violone und „Bombardo“ dagegen im Chorton. ${ }^{8}$ Was im Falle der (als veranschaulichendes Beispiel gewählten) eröffnenden Bassarie für das Ensemble sich als D-Dur zeigte, war für Orgel, Violone und Bombardo C-Dur. Die Entscheidung zwischen Chor- und Kammerton war beim Violone lediglich eine Frage lokaler Üblichkeit, Wechsel vom einen zum anderen Stimmton allenfalls eine Frage der Besaitung. Der „Bombardo“ aber, ein im Folgenden noch näher zu bestimmendes Holzblasinstrument, erweist sich durch seine Chortonstimmung als Instrument einer gegenüber Oboe und „Bassono“ älteren Generation, das, eher überraschend, hier auch unter erheblich veränderten Gegebenheiten des Ensemblemusizierens im Gebrauch bleibt. ${ }^{9}$

Einer generellen Entwicklung im Instrumentenwesen des 16. Jahrhunderts entsprechend waren in dieser Zeit Pommern - gerade gebaute, konisch gebohrte und daher in die Oktave überblasende Doppelrohrinstrumente - zu einer vollständigen „Familie“ ausgebaut worden, über die Michael Praetorius 1619 in Wort und Abbildung sehr detaillierte Auskunft gegeben hat. ${ }^{10}$ Der „Baß-Pommer“ (Abbildung Praet.II, Tafel XI), das Praetorius zufolge

6 Die „historische Aufführungspraxis“ der Gegenwart hat sich für die genannten Stimmtöne auf Standardwerte geeinigt, die zwar die aus vergangenen Epochen überlieferte breite Skala effektiver Stimmton-Werte zu verschleiern geeignet sind, aber einen für Instrumentalisten wie Instrumentenbauer praktikablen Weg eröffnet haben. Diese Standardwerte orientieren sich am Norm-Stimmton neuerer musikalischer Praxis $\left(a^{\prime}=440 \mathrm{~Hz}\right)$, von welchem sie in Halbtonschritten absteigen bzw. aufsteigen. Der Chorton entspricht nach diesem System 466 Hz, der „hohe“ Kammerton 415 Hz, der „tiefe“ 392 Hz. Außerhalb des Systems steht der für das „klassische“ Instrumentarium, d. h. dasjenige der Epoche der Wiener Klassik, Standard gewordene Stimmton $a^{\prime}=430 \mathrm{~Hz}$ - seinerseits eine starke Vereinfachung der vielgestaltigen historischen Realität.

7 Eine Ausnahme bilden Zink und Posaunen, die gleich der Orgel im traditionellen Chorton blieben.

8 Die drei Trompeten können entweder als gemäß heutiger Gepflogenheit transponierend notierte DTrompeten (in der Ausdrucksweise des 18. Jahrhunderts „kammertönige D-Trompeten“) betrachtet werden oder aber als "chortönige C-Trompeten“.

9 Über eine größere Anzahl von (sämtlich aus Danziger Fundus stammenden) Kirchenkompositionen, welche die Verwendung eines „Bombardo“ bezeugen, berichtet Christian Ahrens, Bombart, Bombardo, Bombardone. Bass- bzw. Kontrabasspommer in der Danziger Kirchenmusik des 18. Jahrhunderts, in: Concerto, Nr. 163 (Mai 2001), S. 24-27. Auch Altnickols Osterkantate ist dort, S. 24 rechts, genannt.

10 Syntagmatis musici tomus secundus De organographia, Wolfenbüttel 1619, mit Tafelanhang Theatrum instrumentorum seu Sciagraphia, Wolfenbüttel 1620 (Faksimile-Nachdruck Kassel usw. 1958, = Documenta musicologica I / 14), S. 22, 36 f. und Tafeln VI, XI; Syntagmatis musici tomus tertius, Wolfenbüttel 1619 (Faksimile-Nachdruck Kassel usw. 1958, = Documenta musicologica I / 15), S. 162-168. Im Folgenden sind Teil II bzw. III kurz als „Praet.II“ bzw. „Praet.III“ zitiert, jeweils mit Angabe der Seitenzahl. 
eigentliche, primäre, reguläre Bassinstrument der Familie, der „rechte Baß“ (Praet.II 36), auch „Chorist Baß Pommer“ genannt (III 162)11, italienisch „Bombardo“ (II 36), hatte bei einer Korpuslänge von (gemäß Praetorius’ Abbildung) etwa $180 \mathrm{~cm}$ als „Siebenfingergriff“ $F$, als tiefsten Ton der mit Hilfe dreier zusätzlicher Klappen bewirkten „Extension“ nach unten $C$. Er entsprach damit hinsichtlich seiner Lage dem Hauptinstrument der Fagottfamilie der Zeit, dem „Chorist Fagott" (Praet.II 23 und Tafel X). Dieses im Korpus nur etwa halb so lange, daher ungleich handlichere, außerordentlich verbreitete Instrument unterschied sich vom Basspommer klanglich darin, dass es „stiller und sänffter am Resonantz“ war (II 38), also an Klangkraft unterlegen. ${ }^{12}$

Als obere Umfangsgrenze des Basspommers gibt Praetorius in seiner „Tabella universalis" (II 22) h (als weiße Semibrevis) und $c$ 'an (geschwärzte Semibrevis, Zeichen für Töne, die nicht „ein jeder allezeit [...] assequiren oder erreichen kann“, II 19). ${ }^{13}$ Der resultierende Gesamtumfang $C-c^{\prime}$ stimmt genau mit demjenigen überein, den Ahrens ${ }^{14}$ für die von ihm untersuchten „Bombardo“-Partien ermittelt hat. Gleiches gilt für zwei mit „Bombard o Trombona Grossa“ bzw. „Violon o Bombarde“ bezeichnete Partien aus Kirchenstücken von Dieterich Buxtehude, dem sechschörigen Benedicam Dominum in omni tempore (BuxWV 113) bzw. der Choralbearbeitung Erhalt uns Herr bei deinem Wort (BuxWV 27). ${ }^{15}$ „Bombard“ bzw. „Trombona Grossa“ $(C-c)$ trägt im Zusammenwirken mit (Bass-) „Posauna“ $\left(C-c^{\prime}\right)$ den im Übrigen mit „Trombetta“ I-IV besetzten zweiten Chor. ${ }^{16}$ Der Einsatz des „Bombard“ im Rahmen eines großen Ensembles entspricht dem in unserem Zusammenhang bisher gewonnenen Bild. ${ }^{17}$ Anders, und deshalb von besonderem Interesse, sind die Verhältnisse bei Erhalt uns Herr bei deinem Wort: Zum vierstimmigen Vokalensemble treten hier nur zwei Violinen, „Violon o Bombarde“ und Basso continuo. Der instrumentale Ensemblebass $\left(C-c^{\prime}\right)$, in insgesamt sehr ruhiger Bewegung, gibt nur ganz punktuell das Fortschreiten in Vierteln auf und unterscheidet sich nur minimal vom Basso continuo (Beachtung verdient, dass er in Takt 86-90 und auf der vorletzten Semibrevis eine Oktave unter diesem geführt ist). Offensichtlich erschien der „Bombard“ auch in einer von derje-

11 Der italienische Terminus „,corista“ hat zwar originär mit „coro“, „Chor“ zu tun (und bezeichnet auch den Chorsänger, „Choristen“; daher möglicherweise der verbreitete Irrtum, „Choristfagott“ beziehe sich auf ein Zusammenwirken dieses Instruments mit dem Chorbass). In erweiterter Bedeutung meint er jedoch den Stimmton, Bezugston, und die Mittel (Stimmgabel, Stimmpfeife), ihn anzugeben; ebenso, analog, dasjenige Glied einer Instrumentenfamilie, an dem sich die übrigen im Sinne einer Bezugsgröße orientieren. "Fagotto chorista" findet sich schon bei Lodovico Zacconi, Prattica di musica, Venedig 1592, f. 218 und 218’.

12 In gleichem Sinne äußert sich Georg Falck, Idea boni cantoris, Nürnberg 1688, S. 206: „Fagotto, Dulciano, Dulcin [...] ist etwas stiller am Resonanz als ein Bommart“ (hierzu S. 204: „Bombardo ist ein blasend Instrument, insgemein Bommart genannt").

13 Das „Chorist Fagott" geht dagegen bis $h c^{\prime} d^{\prime}$ (weiße Semibreven) und $e^{\prime} f^{\prime} g^{\prime}(!)$ (geschwärzte Semibreven), II 23.

14 Vgl. Ahrens, Bombart, Bombardo, Bombardone, S. 24 rechts.

15 Das sechschörige Benedicam liegt in einem Faksimiledruck des vollständigen (handschriftlichen) Stimmensatzes der Universitätsbibliothek Uppsala vor (Reihe Autographus Musicus, Stockholm 1973), die Choralbearbeitung innerhalb der Gesamtausgabe (Dietrich Buxtehudes Werke, hrsg. von Hilmar Trede und Dietrich Kilian, Band 8, Leipzig / Hamburg 1958, S. 47 ff.).

16 Besetzung im Übrigen: Chor I Violino 1. Violino 2. Violon.; Chor III fünf als Concertato gekennzeichnete Singstimmen (Sopran I, II, Alt, Tenor, Bass); Chor IV Cornett 1. Cornett 2. Fagotto $\left(C-c^{\prime}\right)$; Chor V Trombona I-III (Schlüsselung: $c 3 / c 4 / f 4$ [Umfang C-h]); Chor VI vier Singstimmen (Sopran, Alt, Tenor, Bass).

17 Vgl. Ahrens, Bombart, Bombardo, Bombardone, S. 24 rechts unten. 
nigen des großen Forte so durchaus verschiedenen Situation als geeignete Alternative zum Violone.

Die bisher referierten bzw. zitierten Beispiele scheinen auf eine allgemeinere Praxis der Zeit hinzudeuten, „Bombardo“-Partien auf den Umfang $C-c$ ' zu beschränken. Das bedeutet freilich nicht, dass höhere Töne als $c$ ' nicht hätten hervorgebracht werden können. Seit jeher lässt sich bei keinem der Überblasung fähigen Instrument die obere Grenze seines Umfangs einheitlich und zuverlässig bestimmen. ${ }^{18}$ Sie ist wesentlich vom jeweiligen Instrument, Rohrblatt bzw. Mundstück und vor allem vom Vermögen des individuellen Musikers abhängig, Die (im Chorton) zweimal, Nr. 3 Takt 55 und 57, bis $d^{\prime}$ geführte „Bombardo“-Stimme von Altnickols Ich lebe und ihr sollt auch leben bietet also keinen Anlass zu besorgten Überlegungen.

Die zum Umfang des Basspommers, eines Instruments der 8'-Lage, so vorzüglich passenden „Bombardo“-Partien, von denen schon die Rede war, eben dem Basspommer zuzuweisen, liegt nahe. Das Nebeneinander von Basspommer und „Bassono“ („Barockfagott“), zweier 8'-Doppelrohrinstrumente also, in einem und demselben Ensemble sollte um so weniger irritieren, als einerseits kraftvolle Verstärkung des Basses zumal in großen Kirchenräumen nur erwünscht sein konnte, andererseits (unabhängig davon, ob die verschiedenen Bassinstrumente gleichlautende Partien spielen oder nicht) es sich um zwei verschiedene Funktionen handelt: die des Basses im instrumentalen Ensemble („Bassono“) und die des Fundaments für das Gesamtensemble, des Basso continuo („Organo“, „Violono“, „Bombardo").

Bei solchen Erwägungen gilt es jedoch, die allem Anschein nach keineswegs unbedeutende Rolle im Blick zu behalten, welche verschiedenen Zeugnissen der Zeit zufolge der um eine Quinte oder Quarte unter dem Basspommer liegende Großbasspommer im 17. und teils bis weit ins 18. Jahrhundert hinein gespielt hat. Michael Praetorius gebraucht für dieses Instrument variierende Benennungen: „Groß Baß Pommer“ (II [13], 22; III 162,164), „Groß Pommer“ (III „142“ [recte 122]), „Dop:[pel] Pom:[mer]“ (III 162), ${ }^{19}$ „Groß Doppel Pommer“ (III 164), „Doppel-Groß Baß Pommert“ (III 163), „Groß Doppel Quint-Pommer" (II Tafel VI); ${ }^{20}$ Praetorius teilt auch die italienische Benennung „Bombardone“ mit. ${ }^{21}$

Schon bei Praetorius lassen sich übrigens, wie dann in aller Folge, Verwischungen des an sich klaren Bedeutungsunterschiedes zwischen „Bombardo“ und „Bombardone“ beobachten. Bei einem zweichörig „mit 4. Posaunen / und 4. Dolcianen oder Fagotten“ zu besetzenden Ensemble werden dem „2. Chorus“ „Fagotti vel Bombardoni“ [statt „Bombardi“] zugewiesen, was nicht etwa vier Großbasspommern meint, sondern vier Glieder der Familie „Bombardo“ in unterschiedlicher Lage und Schlüsselung (Praet.III 165). Analog bezeichnet in einer systematisierenden Instrumenten-,,Tabell“ (III „140“ [recte 120]) „Bombardoni“ die Familie insgesamt, während III 161 in gleichem Sinne „Pommern oder Bombarden“

18 Die (leider nur minimale) praktische Erfahrung des Verfassers mit dem Basspommer hat immerhin den Eindruck wecken können, dass einerseits Töne bis etwa e' / f' durchaus praktikabel sind, andererseits das Instrument insgesamt jedem mit größeren Doppelrohrblättern Vertrauten guten Zugang bietet.

19 „Doppel“ bezieht sich vermutlich, wenn auch hinsichtlich der akustischen Proportion nicht korrekt, auf das Verhältnis der Korpuslängen. Vgl. englisch „Double-bassoon“ als Bezeichnung des Kontrafagotts.

20 "Quint-“ kennzeichnet den Abstand des Instruments zum Basspommer.

21 Entsprechend Georg Falck, Idea boni cantoris, Nürnberg 1688, S. 204: „Bombardone ein grosser Baß Bommart." 
gebraucht wird. Solche Vermischungen sind nicht verwunderlich, wenn man einerseits die häufige Neigung des Sprachgebrauchs zum fülligeren Ausdruck berücksichtigt, andererseits die hinsichtlich baulicher Beschaffenheit indifferente, ausschließlich und in sehr allgemeiner Weise auf den Klangcharakter gerichtete Art der Benennung.

Als [Korpus-]Länge des Großbasspommers gibt Praetorius (II 37) „10. Schuch 1. Zol“ an (etwa $282 \mathrm{~cm}$ ), als Tonumfang Kontra- $F-e$ (weiße Semibrevis) / $f$ (geschwärzte Semibrevis) (II 22); der „Siebenfingergriff“ ergibt Kontra- $B$. Somit entspricht der Großbasspommer in seiner Lage dem „Quint Fagott“ (II 23, 38), dessen Umfang noch um eine geschwärzte Semibrevis $g$ höher reicht. Neben dem „in cantu b molli“ besonders brauchbaren Quintfagott existierte zur Verwendung „in Cantu duro“ das einen Ganzton höhere „Quart Fagott“ (II 23, 38, Abbildung Tafel X); mit dem völlig entsprechenden Umfang Kontra- $G-a$ (II 23). Einen Großbasspommer dieser Lage erwähnt Praetorius nicht, doch könnte ein in Museumsbesitz befindliches Instrument als Zeugnis seiner einstigen Gebräuchlichkeit zu betrachten sein (s.u.). Quint- wie Quartfagott wurden auch „Doppel Fagott“ oder „Fagott grando" [!] genannt (II 23, 38).

Der bei Praetorius (II, Tafel VI) abgebildete „Groß Doppel Quint-Pommer“ hatte nach dem der Tafel beigegebenen Maßstab nicht 10' 1” (s.o.), sondern 10' 5” Korpuslänge, maß also in natura etwa $292 \mathrm{~cm}$. Von vier bisher bekannt gewordenen originalen Großbasspommern, sämtlich in Museumsbesitz ${ }^{22}$, erreicht keiner solche Länge. Drei von ihnen sind annähernd gleich lang (Berlin 271,5 cm; Lübeck $272 \mathrm{~cm}$; Prag 272,5 cm); die Exemplare von Berlin und Lübeck stimmen auch darin überein, dass das dem Zeigefinger der unteren Hand zugeordnete Tonloch (nach üblicher Bezeichnungsweise gegenwärtiger Literatur „IV“) nicht direkt gegriffen wird, sondern mit einer in Ruhelage offenstehenden „Transmissionsklappe“versehen ist. Das Prager Exemplar zeigt an dieser Stelle ein (seinerseits dem Zeigefinger der unteren Hand zugeordnetes) Klappenpaar, das keinesfalls zum originalen Bestand gehören kann, sondern auf einen (vermutlich im 19. Jahrhundert erfolgten) Eingriff zurückgehen muss. ${ }^{23}$ Das in Salzburg befindliche Instrument, mit direkt gegriffenem Tonloch IV und erheblich kürzer $(244 \mathrm{~cm})$, könnte einen „Groß Doppel Quart-Pommer“ mit „Siebenfingergriff“ $C$ und tiefstem Ton Kontra- $G$ darstellen. ${ }^{24}$

22 1. Berlin, Staatliches Institut für Musikforschung Stiftung Preußischer Kulturbesitz, Musikinstrumenten-Museum, Inv. Nr. 289. Vgl. Curt Sachs, Sammlung alter Musikinstrumente bei der Staatlichen Hochschule für Musik zu Berlin. Beschreibender Katalog, Berlin 1922, Sp. 272, Abbildung Tafel 27. Das Instrument stammt aus der Danziger Marienkirche. - 2. Lübeck, St. Annen-Museum. Vgl. Georg Karstädt, Die Sammlung alter Musikinstrumente im St. Annen-Museum, o. O. u. J., unpaginiert (= Lübecker Museumshefte, 2), S. [4], [9], Abbildung S. [13]. Das Instrument wurde 1685, in der Amtszeit Dietrich Buxtehudes, für die Marienkirche angekauft (vgl. Kerala J. Snyder, Dieterich Buxtehude, Organist in Lübeck, New York / London 1987, S. 375, 378 f.); um diesen „Bombard“ [!] „Orgelmässig zu bringen“, d. h. an den Stimmton der Orgel anzugleichen, musste „ein newes Messings Es oder Rohr" angefertigt werden (S. 476). - 3. Prag, Narodni Museum, Inv.-Nr. 480 E. Vgl. Otto Oromszegi, The Bombards of Master „W" of Rozmberk, in: The Galpin Society Journal 21, 1968, S. 97-104; Abbildung Plate IX(b). - 4. Salzburg, Museum Carolino Augusteum, Verzeichnis Birsak, Familie 12, Nr. 8 (Geir. 187). Vgl. Kurt Birsak, Die Holzblasinstrumente im Salzburger Museum Carolino Augusteum. Verzeichnis und entwicklungsgeschichtliche Untersuchungen, in: Jahresschrift Salzburger Museum Carolino Augusteum 18 (1972), Salzburg 1973, S. 34, 102-104, 138; Abbildung Tafel V.

23 Vgl. Oromszegi (wie Anm. 22), S. 102.

24 Die Ermittlung des originären Stimmtons historischer Doppelrohrinstrumente ist deshalb in besonderem Grade schwierig und unsicher, weil, selbst abgesehen von dem ebenso erheblichen wie unvermeidlichen Einfluss des jeweiligen Doppelrohrblatts („Rohrs“), das auf das akustische Verhalten des Instruments womöglich noch stärker einwirkende Mundrohr („S“, „S-Bogen“), falls überhaupt 
Mit einem Umfang von etwa zwei Oktaven auf Basis Kontra- $F$ oder Kontra- $G$ steht der Großbasspommer, gleich dem Quint- und Quartfagott und auch dem im deutschsprachigen Raum vom 17. bis Ende des ersten Drittels des 18. Jahrhunderts vielfach belegten sechssaitigen Violone auf Kontra- $G$, zwischen Bass- und Kontrabasslage. Eine in der Musik jenes Zeitraums regulär zu nennende, von $C$ aus etwa zwei Oktaven durchlaufende Basspartie konnten die genannten Instrumente in der notierten Lage (der „Achtfußlage“) ausführen, wobei unter Umständen einige der oberen Töne nach unten zu oktavieren waren; ${ }^{25}$ sie konnten aber auch, indem sie den Bereich des notierten $C-E$ bzw. $C-F$ is nach oben oktavierten, als „Sechzehnfuß“-Instrumente eingesetzt werden, deren erklingende Töne um eine Oktave unter den notierten lagen. Wie ein solches Verfahren im Einzelnen angewandt wurde, war zweifellos ebenso eine Frage jeweiliger täglicher Praxis wie der noch heute erforderliche Umgang von Spielern des viersaitigen Kontrabasses (auf Kontra-E) mit dem notierten Bereich $C$-Dis/Es. Mit welcher Selbstverständlichkeit es akzeptiert wurde, veranschaulicht am besten der bis in das 20. Jahrhundert hinein belegte Gebrauch dreisaitiger Kontrabässe auf Basis Kontra- $G$ (mit Quintstimmung) oder sogar Kontra- $A$ (mit Quartstimmung). ${ }^{26}$

Über den „brummenden Violone“, von „wichtiger“ „Fundament“-Funktion „zu vollstimmigen Sachen / als Chören und dergleichen / nicht weniger auch zu Arien und so gar zum Recitativ“, teilt Johann Mattheson 1713 mit, sein „Thon“ sei „sechzehnfüßig“, leider ohne Angaben zu Saitenzahl und Stimmung. ${ }^{27}$ Matthesons Ausführungen kehren in weitgehend wörtlicher Übernahme 1732 bei Joseph Friederich Bernhard Caspar Majer wieder, werden dort aber mit dem sechssaitigen Violone der Stimmung Kontra- $G, C, F, A, d, g$ in Verbindung gebracht. ${ }^{28}$ Dies mag als bloße Folge der Kompilation zu betrachten sein; doch

vorhanden, kaum je als authentisch zugehörig gesichert ist (vgl. hierzu auch die Anm. 22 unter 2.). Angaben der Literatur zum Stimmton sollten daher, sofern nicht hinlänglich dokumentiert, stets mit Vorbehalt aufgenommen werden.

25 Ein von Guntram Wolf, Kronach, nach einem Original des Kunsthistorischen Museums Wien (Inv.Nr. C.198) gebauter „Quartbass-Dulzian“ (nach Praetorius' Benennung ein „Quart Fagott“) im Besitz des Verfassers lässt sich mühelos und zuverlässig bis (klingend) $d$ ' und es' blasen. Als in diesem Zusammenhang aufschlussreich erweist sich eine aus Stift Kremsmünster (Oberösterreich) stammende Canzon a 6 Voc. Duobus cho: Tre Violini é Tre Fagotti von J. Lechler, 1645, die dem Verfasser in einer handschriftlichen Spartierung unbekannten Urhebers vorliegt. Der Benennung der einzelnen Stimmen zufolge ist sie im oberen, hoch liegenden Chor mit „Violin“ (g2), „Violin“ (g2) und „Viola da Bracio“ (c3) besetzt, im unteren mit „Fagott“ (f4, Umfang $c-c^{\prime}$ ), „Fagott“ (f4, H-c') und „Fagotto grosso“ “ $\left(\mathrm{f} 4, C-c^{\prime}\right)$. Insgesamt in sehr ruhiger Bewegung gehalten, weist sie unerwarteterweise ausgerechnet der Viola da braccio und, besonders, dem „Fagotto grosso“ (aller Wahrscheinlichkeit nach einem „Quartbass") fast virtuose Diminutionsfiguren zu, die den ganzen angegebenen Umfang in Anspruch nehmen und zweimal bis $c^{\prime}$ aufsteigen. Nach dem im Ensemble gewonnenen Eindruck des Verfassers ist der Klangcharakter des Dulzians auf Kontra- $G$ deutlich der eines tiefen Bass-, nicht eines Kontrabass-Instruments.

26 Noch Richard Strauss befürwortet in seinen Anmerkungen zu Berlioz' Instrumentationslehre (Instrumentationslehre von Hector Berlioz. Ergänzt und revidiert von Richard Strauss, Leipzig 1905, S. 105 links) die Verwendung des „italienischen drei-saitigen [Kontrabasses], der sich unvergleichlich besser zur Kantilene eignet" in Verbindung mit dem viersaitigen.

27 Das Neu=Eröffnete Orchestre[...], Hamburg 1713 (Faksimile-Nachdruck Hildesheim usw. 1993), S. $285 \mathrm{f}$.

28 Museum musicum [...] das ist / Neu=eröfneter Theoretisch= und Practischer Music-Saal [...], Schwäbisch Hall 1732 (Faksimile-Neudruck Kassel und Basel 1954, = Documenta musicologica I / 8), S. 80; zweite (im vorliegenden Zusammenhang unveränderte) Auflage unter dem Titel Neu=eröffneter 
hatte Majer offensichtlich keine Bedenken, ein in der Tiefe bei Kontra- $G$ endendes - in der Ausdrucksweise der Zeit „Zwölffüßiges“ - Instrument „16.füßig“ zu nennen.

Noch entschiedener wird von Martin Heinrich Fuhrmann im Jahre 1706 der „Bombardone“ als Repräsentant der 16'-Lage im großen Kirchenraum hervorgehoben: „1. Bombardone, der Baß-Bommert / dicitur a Bombo seu a Bombarda, weil er einen gravitätischen 16füßigen Thon gleich einem Posaun-Baß in der Orgel starck brummet und knallet. Ist ein herrlich Instrument pro Fundamento, und nur zu bedauren / daß es heute so in Abgang gebracht und davor die 8. füßige Frantz. Bassons wieder auffgebracht worden / welche in einem Zimmer zwar starck genug thönen / aber in einer grossen Kirchen nicht durchdringen. Denn kein 8füßiger Thon dringt mit Nachdruck in einer grossen Gemeine durch / weil eine Menschen Stimme eben so tieff gehet; Aber ein 16füßiger Thon Z.E. nur ein 16füßiger schwacher Sub-Baß dringet durch etliche 1000. Leute durch / weil er eine Octav unter eines Menschen Tieffe gehet.“ 29 Fuhrmann berichtet anschließend über „eine starcke Figural Music in einer grossen Kirchen / so gerüttelt und geschüttelt voll Menschen war“, wobei „aus gewissen Ursachen“ die Orgel nicht beteiligt war und zum Ausgleich „gerne ein Dutzend Frantzö[s]ische Baß Geigen [basses de violon] zum Fundament gestrichen [wurde] / welche noch wol ein halb Dutzend Frantzö[s]ische Fagottisten secundirte. Allein wie kräfftig ja vielmehr / wie gedämpfft dieses weitläufftigte Frantzö[s]ische Fundament von ferne thönete / höreten die Auditores mit Verwunderung am besten; Ja ich meyne / hätte der eintzige gravitätische Baß-Bommert mit seinem 16füßigen Thon allhier nicht nachdrücklich durchgedrungen / und allen Fundament Mangel ersetzet, so wäre diese schöne Music schier ohne Fundament gewesen." Im nächsten Absatz weist Fuhrmann indirekt nochmals auf das besondere Durchsetzungsvermögen des „Bombardone“ hin: „2. Fagotto seu Dolciano ein 8füßiger Dulcian ist Chor-Thon. Bassone [!], ein Frantzö[s]ischer Fagott aber Cammer-Thon / thönet gelinder als ein Baß-Bombard.“

Alles, was Fuhrmann über dieses Instrument sagt, kann ohne Vorbehalt auf den Großbasspommer mit tiefstem Ton Kontra- $F$ bezogen werden - wofür ja auch dessen Verwendung in den Hauptkirchen von Danzig und Lübeck spricht (vgl. Anm. 22). Weitere historisch bezeugte Gegebenheiten laden jedoch dazu ein, den Blick noch in eine andere Richtung zu lenken. Im Jahre 1671 lieferte ein Instrumentenmacher namens Johannes Bohlmann (auch Pohlmann, Boleman, Bollmann) von Frankenhausen (Thüringen) für die beiden Leipziger Hauptkirchen St. Thomae und St. Nicolai je einen „Octav Bombart“ 30 Das Instrument der Thomaskirche erscheint in einem Inventarverzeichnis vom Jahre 1789 als „Großer Bombard, so nicht gebraucht wird“. 31 „Octav“ meint demnach, da es sich offensichtlich um ein auffällig großes Instrument handelte, ein Unteroktavverhältnis zu einem Bezugsinstrument - zweifellos einem der Basslage, im Falle des „Bombart“ im primären Sinne also dem Basspommer mit tiefstem Ton $C$.

Ein bis Kontra- $C$ reichender Pommer wäre aber, im Unterquartverhältnis $4: 3$ zum $271,5 \mathrm{~cm}$ langen Berliner Großbasspommer, etwa $360 \mathrm{~cm}$ lang und damit wohl für Hersteller wie Spieler außerhalb aller Praktikabilität. Nur eine das Korpusmaß auf ungefähr die

Theoretisch $=$ und Pracktischer Music=Saal [...], Nürnberg 1741 (Reprint o. O. u. J. [Michaelstein/ Blankenburg 1990]), S. 100.

29 Musicalischer Trichter [...], „Franckfurt an der Spree“ [Berlin] 1706, S. 91 f.

30 Vgl. Arnold Schering, Die Leipziger Ratsmusik von 1650 bis 1775, in: Archiv für Musikwissenschaft 3, 1921, S. 35; ferner Herbert Heyde, Contrabassoons in the 17th and early 18th century, in: The Galpin Society Journal 40, 1987, S. 26.

31 Vgl. Heyde, Contrabassoons in the 17th and early 18th century, S. 26. 
Hälfte der akustisch geforderten Bohrungslänge reduzierende Konstruktion, die Fagottbauweise also, ermöglicht praktikable Instrumente von so tiefer Lage.

Der „grosse Fagotcontra / welcher noch ein Quart unter dem DoppelFagott / unnd also ein Octav unterm ChoristFagott / das C von sechtzehen Fueß Thon geben unnd intoniren sol", von dem Praetorius (II 38) als in Arbeit befindlich berichtet, existierte andernorts längst vor 1619 und war wohl schon im 16. Jahrhundert entstanden. ${ }^{32}$ Zwei gleichartige, späte Exemplare des Kontrabassdulzians, knapp $200 \mathrm{~cm}$ lang, einer von ihnen datiert 1681, haben sich im Schlossmuseum Sondershausen (Thüringen) erhalten. ${ }^{33}$ Sie tragen als Brandstempel ein Monogramm, welches Heyde ${ }^{34}$ überzeugend als J B, Johannes Bohlmann, interpretiert, welches demnach die Sondershausener Kontrabassdulziane als Arbeit des Urhebers der nach Leipzig gelieferten „Octav Bombarte“ ausweist. „Zwene grosse BassBommerte" enthält ein um 1714 aufgezeichnetes Inventarium, Über die, zur Hochgräffichen Rudolstädtischen Hoff-Capell gehörigen Musicalischen Sachen und Instrumente zusammen mit „Zwene Französische Bassons" und einem „8tav Fagott“, das Heyde zweifellos zutreffend als Kontrafagott der neueren, „barocken“ Art definiert. ${ }^{35}$

Nicht beweisbar zwar, aber doch $\mathrm{m}$. E. wahrscheinlich gemacht wird durch die mitgeteilten Gegebenheiten, dass Martin Heinrich Fuhrmann und seine Zeit des späten 17. und der ersten Hälfte des 18. Jahrhunderts mit „Bombardone“, „Bombardo“, „Baß-Bombard“, „Octav Bombart“, „Großer Baß-Bommert“ a u ch den in der Tat „sechzehnfüßigen“ Kontrabassdulzian meinen konnte, zumal angesichts eines schon bei Praetorius konstatierten unscharfen Sprachgebrauchs (s. o.). ${ }^{36}$ Unser modernes Bedürfnis nach Eindeutigkeit und Einheitlichkeit in solchen Dingen wird dadurch gewiss nicht befriedigt; dafür aber ein Blick in die Realität handwerklichen Umgangs mit Benennungen und in die Vielfalt musikalischer, aufführungspraktischer Möglichkeiten eröffnet.

Das Nebeneinander von zwei „großen Baß-Bommerten“ und einem „Oktavfagott“ im zuvor zitierten Rudolstadter Inventar des frühen 18. Jahrhunderts wiederholt sich, ebenso konkret wie aufschlussreich, in dem seinerseits auf eine Hofkapelle zurückgehenden Instrumentenfundus des Schlossmuseums zu Sondershausen. Außer den beiden oben erwähnten Kontrabassdulzianen von Johannes Bohlmann (Inv.-Nr. Mu 1 bzw. Mu 38) findet sich dort ein „barockes“ Kontrafagott mit drei Klappen (Mu 2), unsigniert, jedoch wie ein Zwilling dem im Besitz des Musikinstrumenten-Museums der Universität Leipzig (Inv.-Nr. 3394) befindlichen, signierten und datierten Instrument von Andreas Eichentopf, Nordhausen 1714, gleichend. (Auch ein „französischer Basson“ von Richard Haka, Amsterdam, ist unter der Inv.-Nr. Mu 5 vorhanden.) ${ }^{37}$

32 Ebd., S. $33 \mathrm{f}$.

33 Vgl. ebd., S.24 f., 26 und Plate I. Ein Nachbau eines dieser Instrumente aus der Werkstatt von Guntram Wolf, Kronach, im Besitz des Verfassers, hat sich im Rahmen eines größeren vokal-instrumentalen, Zinken und Posaunen einschließenden Ensembles in Verbindung mit einem Violone als tragfähiges Fundament erwiesen; vgl. CD Michael Praetorius, Weihnachtliche Motetten. Choralkonzerte, Hassler Consort Franz Raml (MDG 614 0660-2, 1996).

34 Ebd., S. 27.

35 Ebd., S. 32.

36 Wie weit diese Unschärfe gehen kann, zeigt Johann Philipp Eisel, Musicus autodidaktos, Erfurt 1738, der (S. 104) in der Formulierung „Die Teutschen Bassons, Fagotte, oder Bombardi, wie sie unsre Teutsche Vorfahren [...] geführet / sind nicht mehr im Gebrauch [...]“ „Teutscher Basson“ („deutsch“ im Gegensatz zum „Basson“ schlechthin, dem modernen Instrument französischer Art), „Fagott“ und „Bombardo“ als synonyme Bezeichnungen einer und derselben Sache erscheinen lässt.

37 Vgl. Heyde, Contrabassoons in the 17th and early 18th century, insbesondere Plate I. 
Die „Bombardo“-Stimmen Buxtehudes und der Danziger Kirchenpraxis in Verbindung mit solchen Kontrafagotten als, sozusagen Vertretern einer nächsten instrumentalen Generation lassen auf eine Kontinuität des Gebrauchs solcher am Basso continuo beteiligten Fundament-Instrumente im Rahmen größerer und großer Ensembles schließen. ${ }^{38}$ In dieser Kontinuität steht auch der „Bassono grosso“, für welchen J. S. Bach 1749, anlässlich der letzten Wiederaufführung seiner Johannes-Passion (mit stärker besetztem Orchester), eine seit 1724 vorhandene Basso-continuo-Stimme einrichtete ${ }^{39}$ - wie zu erwarten, überwiegend durch „tacet“-Vermerke. Der „Bassono grosso“ wirkt in allen Chören und Chorälen mit, darüber hinaus in den letzten drei Takten (!) der Tenorarie Nr. 13, in den Ritornellen der Bassarie Nr. 24, im Vivace-Teil der Altarie Nr. 30 (mit Anweisung „Tutti mà piano“ in der „Bassono grosso“-Stimme) und sogar - auf den ersten Blick überraschend, ja irritierend, doch, wie inzwischen durch die Erfahrung zahlreicher Aufführungen bestätigt, mit vorzüglicher Wirkung - im Bass-Arioso Nr. 19 (mit Anweisung „Arioso e pianissimo“). ${ }^{40}$ Der Umfang der Partie, $C$-es', kann nur einem als Kontrafagott mit tiefstem Ton Subkontra- $B$ definierten „Bassono grosso“ sinnvoll zugeordnet werden. Er wäre, von einem Quart- oder Quintfagott „barocker“ Art (tiefster Ton Kontra-Fbzw. Kontra-Es) in 16'-Lage ausgeführt, ständig im Bereich der unbequem zu greifenden tiefsten Töne (ganz abgesehen vom Verzicht auf Kontra- $C$ bis Kontra- $E$ bzw. Kontra- $C$ bis Kontra- $D$ ); in der notierten (8'-) Lage aber extrem hoch.

Bachs beschriebene Verwendung eines Kontrafagotts in der Johannes-Passion als instrumentatorische Besonderheit oder vollends als „Einfall“ zu betrachten, wäre zweifellos verfehlt. Eine Besonderheit im Sinne eines freundlichen Zufalls ist eher, dass er die originär vorhandene Stimmbezeichnung „Continuo“ eigenhändig durch die Worte „pro Bassono grosso" ergänzte - auch ohne solchen Zusatz hätte die Stimme ihren Dienst nicht weniger zufriedenstellend getan. Vielmehr sollte hier ein weiteres Zeugnis für Bachs deutliche Tendenz zur klanglichen „Gravität“ gesehen und die, schon durch die Existenz so außerordentlich mühsam herzustellender Instrumente allein nahegelegte, Möglichkeit ins Auge gefasst werden, der „Bassono grosso“ sei auch sonst des Öfteren an der Ausführung des Basso continuo beteiligt gewesen.

Einen frühen Nachweis der genannten Tendenz liefert Bachs Entwurf für Umbau und Erweiterung der Orgel in der Kirche Divi Blasii zu Mühlhausen (Thüringen) aus dem Jahre 1707. ${ }^{41}$ Im Pedal sollte der Posaunenbass (16') mit neuen, größeren Aufsätzen und veränderten Kehlen versehen werden, um „eine viel beßere gravität“ zu erlangen, ferner ein Untersatz 32' auf eigener Windlade neu hinzugefügt werden, „welcher dem ganzen Wercke die beste gravität giebet“. Im Hauptwerk („Obermanual“) sollte „Trompette“ (8') - ein doch keineswegs unwichtiges Register - einem „Fagotto 16.f[uß] thon“ weichen, „welcher zu allerhandt neuen inventionibus dienlich, und in die Music sehr delicat klinget“: Bemerkenswert erscheint die Bezugnahme auf (figurale) „Music“, d. h. auf das Zusammenwirken der Orgel mit dem vokalen und instrumentalen Ensemble. Möglicherweise sollte das sechzehnfüßige „Rohrwerk“, abgesehen von seinem unbezweifelbaren Beitrag zur Gravität des

38 Ahrens, Bombart, Bombardo, Bombardone, S. 24 rechts, weist auf den lokalgeschichtlich nachgewiesenen Einsatz eines Basspommers (!) bei einer 1823 erfolgten Aufführung des Oratoriums Das Weltgericht von Johann Christian Friedrich Schneider (1786 - 1853) hin.

39 Vgl. Hans-Joachim Schulze und Christoph Wolff, Bach-Compendium, Band 1, Teil 3: Vokalwerke III, Leipzig / Frankfurt am Main 1988, S. 991.

40 Nummerierung nach der Edition der Neuen Bach-Ausgabe (NBA) II/4.

41 Abdruck bei Philipp Spitta, Johann Sebastian Bach, Erster Band, Leipzig 1873, S. 351-353. 
Orgelwerks insgesamt, auch in Vertretung eines Doppelrohrinstruments der 16'-Lage nützlich werden. Parallele Fälle von Manual-Rohrwerken zu 16' sind nicht schwer zu finden. So besaß die 1734-1738 von Johann Friedrich Wender und Sohn für die „oberstädtische Hauptkirche Beatae Mariae Virginis" desselben Mühlhausen erbaute Orgel im Hauptwerk einen „Basson durchs ganze Clavier“ 16. ${ }^{42}$ Die Merseburger Domorgel enthielt im Hauptwerk („Großmanual“) „Trompete oder Bombart“ 16’, im Rückpositiv „Fagott“ 16’; ganz ähnlich die 1743 von Zacharias Hildebrandt erbaute Orgel von Naumburg, St. Wenzel, im Hauptwerk „Bombart“ 16’ (über den ganzen Manualumfang $C-c^{3}$ ), im Rückpositiv „Fagott“ 16. ${ }^{43}$ Johann Christoph Wiegleb schließlich gab dem Oberwerk („2. Clavier“) seiner 1738 vollendeten Orgel für die Stiftskirche St. Gumbertus zu Ansbach ein von $C-c^{3}$ laufendes Rohrwerk zu 16', das in den Quellen teils „Fagott“, teils „Sordün“, teils „Fagottbaß“" genannt wird. ${ }^{44}$

In geänderter Form, in der Tiefe nur noch bis Kontra- $D$ oder maximal bis Kontra- $C$ is reichend, dadurch um rund einen Meter kürzer geworden als sein Vorgänger „barocker“ Art und um vieles handlicher, behielt das Kontrafagott in der Epoche der Wiener Klassik als Fundament-Bass im groß besetzten Orchester wie in der „Harmoniemusik“, den nur mit Bläsern besetzten Formationen, eine gewisse Bedeutung. Im Orchester der Romantik vernachlässigte man es zusehends. ${ }^{45}$ Das mit der Tendenz zu stärkerer Streicherbesetzung und Vermehrung der Blechbläser wachsende Interesse an voluminösen, tonstarken Blasinstrumenten tiefer Lage konzentrierte sich auf solche mit Kessel- bzw. Trichtermundstück. Gegenüber der Fähigkeit zur Kraftentfaltung trat dabei der spezifische Klangcharakter, ja sogar die Oktavlage in den Hintergrund: Serpent, Basshorn, Ophicléide, partiell auch die Tuba gehören der 8'-Lage, nicht der 16'-Lage an. Sehr bezeichnend für die Situation der Zeit ist, dass „Bombardone“, die alte Bezeichnung des Großbasspommers (oder, unscharf verwendet, tiefer Pommern überhaupt, jedenfalls aber von Doppelrohrinstrumenten), nunmehr Ophicléide oder tiefe „Ventilbügelhörner“ („Bombardons“) meinte. Erst im späten 19. Jahrhundert erlangte, neben der schon länger heimisch gewordenen Tuba, durch Richard Wagners Parsifal, durch Kompositionen von Johannes Brahms und vor allem Richard Strauss das Kontrafagott erneut Bedeutung und einen festen Platz im „großen“ Orchester. Eine Erscheinung neuester Zeit ist, dass der sich ausbreitende professionelle Umgang mit Instrumenten älterer auch das Kontrafagott in seinen auf dem Weg durch die Musikgeschichte sich verändernden Gestalten erfasst hat.

42 Jakob Adlung, Musica mechanica organoedi, Berlin 1768 (originalgetreuer Neudruck, hrsg. v. Christhard Mahrenholz, Kassel 1931), S. 259 f. Das anscheinend in besonderem Ausmaß für Figuralmusik in Anspruch genommene Orgelwerk hatte eine höchst ungewöhnliche Einrichtung, „Zwei Kammerkoppel, eins Groß= das andere Kleinkammerton, durchs ganze Werk“, d. h. Transpositionszüge zur Verwandlung des (primären) Chortons in sei es den hohen („Kleinkammerton“), sei es den tiefen Kammerton („Großkammerton“). Ob „durchs ganze Werk“ wörtlich als Ausdehnung der Transposition auf alle drei Manualwerke und das Pedal genommen werden kann oder aber nur das „Werk“ im älteren, engeren Sinne des Hauptwerks meint, steht dahin.

43 Adlung, Musica mechanica organoedi, S. 255 f. (Merseburg) bzw. 263 f. (Naumburg).

44 Vgl. Egert Pöhlmann, Die Quellen für Johann Christoph Wieglebs Stiftskirchenorgel von 1738 in Ansbach-St. Gumbertus, in: Almanach der Bachwoche Ansbach 1989, S. 86, 88, 95.

45 Vgl. des Verfassers Beitrag Im Abendrot, in: Compositionswissenschaft. Festschrift Reinhold und Roswitha Schlötterer zum 70. Geburtstag, hrsg. von Bernd Edelmann und Sabine Kurth, Augsburg 1999, S. $284-$ 286 (Exkurs: Zum Kontrafagott bei Richard Wagner). 